\title{
Correction to: Recent advances in CRISPR technologies for genome editing
}

\author{
Myeonghoon Song ${ }^{1}$ Taeyoung Koo ${ }^{1,2,3}$
}

Accepted: 26 July 2021 / Published online: 21 August 2021

(C) The Pharmaceutical Society of Korea 2021

Correction to: Arch. Pharm. Res. (2021) 44:537-552 https://doi.org/10.1007/s12272-021-01336-4

In the original publication of the article, the Fig. $2 \mathrm{~d}$ was erroneously published. The corrected version (Fig. 2d) is given below:

The original article has been corrected.

The original article can be found online at https://doi.org/10. 1007/s12272-021-01336-4.

Taeyoung Koo taeyoungkoo@khu.ac.kr

1 Department of Life and Nanopharmaceutical Sciences, College of Pharmacy, Kyung Hee University, Seoul, Republic of Korea

2 Department of Biomedical and Pharmaceutical Sciences, College of Pharmacy, Kyung Hee University, Seoul, Republic of Korea

3 Department of Pharmaceutical Science, College of Pharmacy, Kyung Hee University, Seoul 02447, Republic of Korea 
a
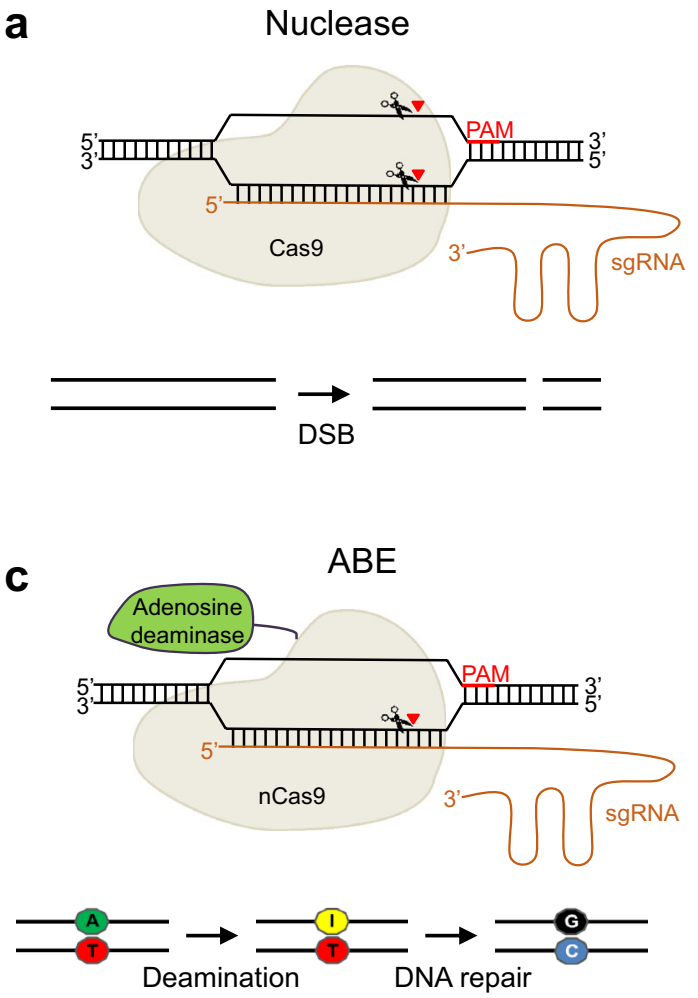

e
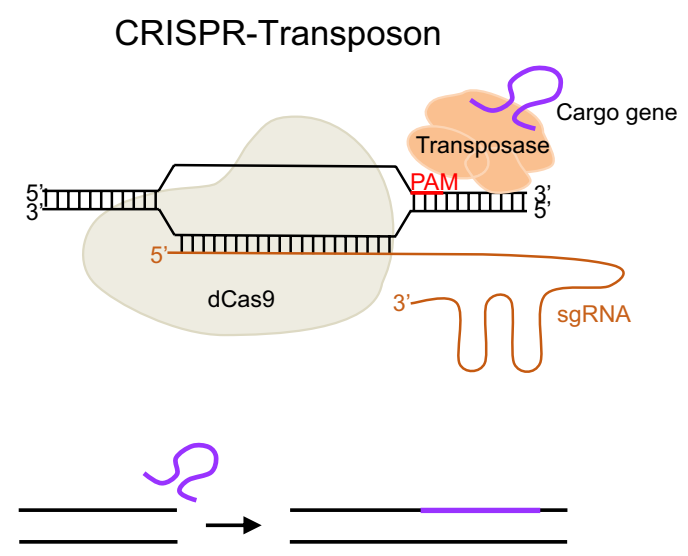

Cargo gene insertion
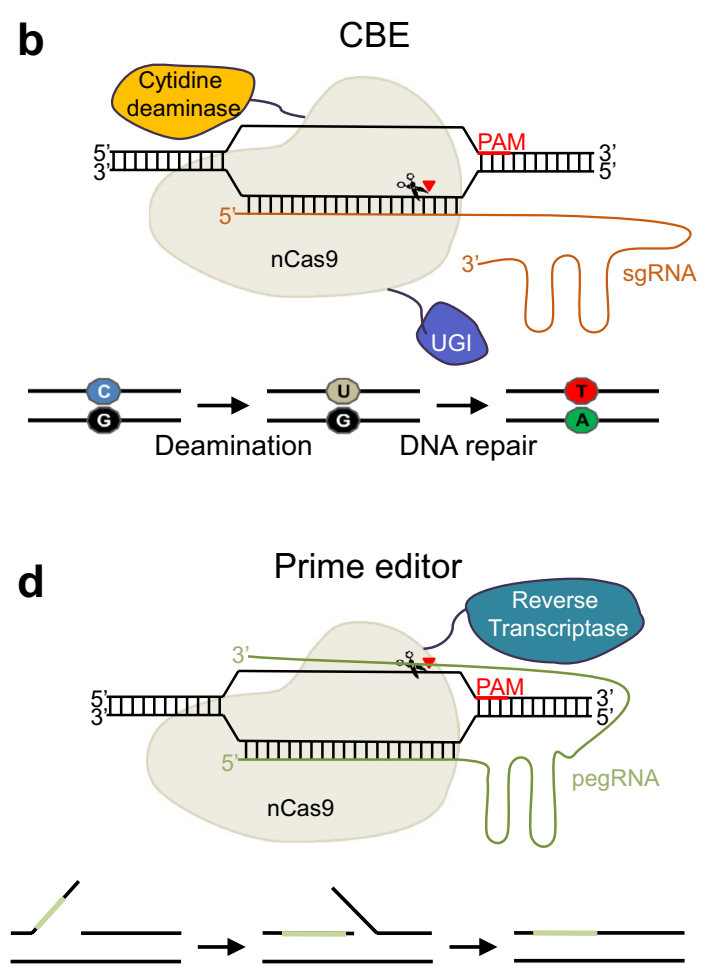

Flap equilibration Desired editing
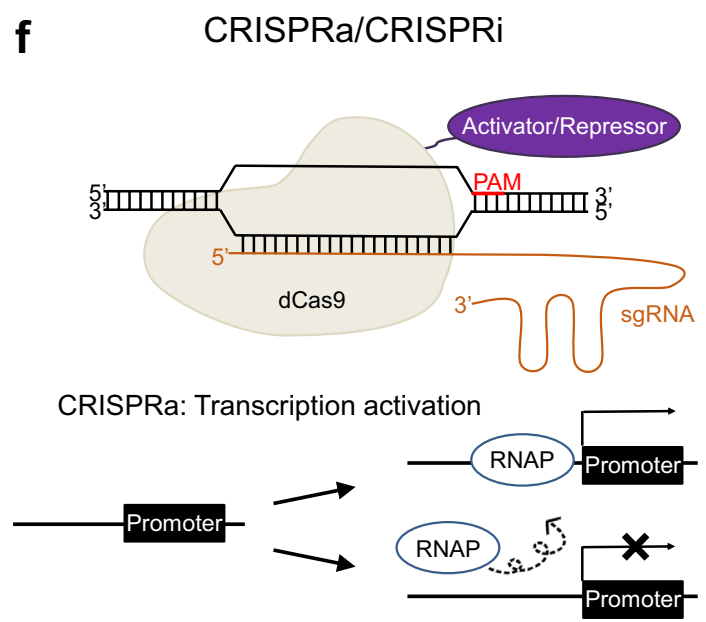

CRISPRi: Transcription repression

Fig. 2 Schematic summary of CRISPR/Cas tools used for genome editing. a The CRISPR/Cas9 system recognizes a PAM and generates DSBs at a site $3 \mathrm{bp}$ upstream from the PAM. b CBE consists of an inactive or nickase form of Cas9 (dCas9 or nCas9) fused to a cytidine deaminase and UGI. After the dCas9 or nCas9 domain of a CBE recognizes a specific sequence, the cytosine deaminase deaminates C to generate U. Then, the $\mathrm{G}$ in the opposite strand is converted to A by cellular mismatch repair, and $\mathrm{C}$ is converted to T. UGI prevents the U from undergoing cellular base excision repair. c ABEs are constructed by fusion of nCas9 to E. coli tRNA adenosine deaminase, which deaminates A to generate I, which is then converted to G by DNA repair or replication. $\mathbf{d}$ Prime editors are constructed by fusion of an engineered reverse transcriptase domain to nCas9. A pegRNA binds to the 3' end of the exposed target DNA strand, which was generated by nCas9. Then, the desired gene edit (which is contained in the pegRNA) is incorporated into the DNA by reverse transcriptase. e The Cas-transposon system was developed by fusing a transposase to Cas9. After the targeted sequence is recognized by Cas9 (dCas9, Cas12k or Cascades), the transpose inserts the desired sequence at the site. $\mathbf{f}$ Tools for CRISPR/Cas-mediated epigenetic regulation are constructed by conjugation of transcriptional repressors or activators to dCas9, generating CRISPRa and CRISPRi systems, respectively. In CRISPRa systems, transcriptional activators recruit RNA polymerase and transcription factors to a promoter and promote transcription. In CRISPRi systems, transcriptional repressors prevent binding of RNA polymerase (RNAP) to the promoter of interest 
Publisher's Note Springer Nature remains neutral with regard to jurisdictional claims in published maps and institutional affiliations. 